\title{
Cancer communication research in the era of genomics and precision medicine: a scoping review
}

\author{
Kimberly A. Kaphingst, ScD ${ }^{1,2}$, Emily Peterson, $\mathrm{PhD}^{3}$, Jingsong Zhao, MPH ${ }^{1}$, Anna Gaysynsky, $\mathrm{MPH}^{4}$, \\ Ashley Elrick, $\mathrm{MA}^{2}$, Soo Jung Hong, $\mathrm{PhD}^{5}$, Melinda Krakow, $\mathrm{PhD}^{3}$, Manusheela Pokharel, $\mathrm{MS}^{2}$, \\ Chelsea L. Ratcliff, MA ${ }^{2}$, William M. P. Klein, PhD ${ }^{3}$, Muin J. Khoury, MD, PhD $^{6}$ and \\ Wen-Ying Sylvia Chou, $\mathrm{PhD}, \mathrm{MPH}^{3}$
}

Effective use of genetic and genomic data in cancer prevention and treatment depends on adequate communication with patients and the public. Although relevant empirical work has emerged, the scope and outcomes of this communication research have not been characterized. We conducted a comprehensive scoping review of recent published research (2010-2017) on communication of cancer-related genetic and genomic testing (CGT) information. Searches in six databases revealed 9243 unique records; 513 papers were included. Most papers utilized an observational quantitative design; fewer utilized an experimental design. More attention has been paid to outcomes of CGT results disclosure than to decision making regarding CGT uptake or the process of results disclosure. Psychosocial outcomes were most common across studies. This literature has a strong focus on $B R C A 1 / 2$, with few papers focused on Lynch syndrome or next-generation technologies. Women,
Caucasians, older adults, and those of higher socioeconomic status were overrepresented. Research gaps identified include the need for studies on the process of CGT communication; examining behavioral, decision making, and communication outcomes; and inclusion of diverse populations. Addressing these gaps can help improve the use of genomics in cancer control and reduce disparities in access to and use of CGT.

Genetics in Medicine (2019) 21:1691-1698; https://doi.org/10.1038/s41436018-0402-0

Keywords: communication; cancer; genetic testing; return of results; decision making

\section{INTRODUCTION}

Advances in genetic and genomic technologies are transforming cancer prevention and care. ${ }^{1}$ Genetic-based risk assessment can help define cancer risks for individuals and families and facilitate decision making about risk management options. $^{2-6}$ Genomic tumor data may also inform choice of therapy for cancer patients. ${ }^{7}$ Effective use of genetic and genomic data in cancer care depends strongly upon communication with patients and the general public. Given that genetic and genomic information generated for individuals is becoming increasingly complex, understanding how this information is communicated and understood is a key priority for research and clinical practice.

A growing body of empirical literature has emerged to address these questions. Yet the scope and nature of this work are diffuse. A comprehensive examination of the state of the science on communication of cancer-related genetic and genomic testing (hereafter CGT) will add to our understanding of which communication processes and outcomes have been the focus of previous studies and identify research gaps. Prior reviews have examined some aspects of the literature on communication about CGT and have mostly focused on the psychosocial and behavioral outcomes of returning genetic test results in the context of inherited cancer syndromes. ${ }^{8-10}$ Other reviews have focused specifically on lower risk contexts. For example, Hollands et al. ${ }^{11}$ examined the impact of communicating DNA-based disease risk estimates on risk-reducing health behaviors and behavioral motivation, ${ }^{11}$ and McBride et al. ${ }^{12}$ reviewed consumers' views on direct-to-consumer genetic information. ${ }^{12}$ Our recent landscape analysis of published reviews and meta-analyses found that the most frequent area of focus among these reviews was the psychological and behavioral impact of testing. ${ }^{13}$ However, the landscape analysis did not examine or synthesize the characteristics of the individual papers that comprise this literature.

\footnotetext{
${ }^{1}$ Huntsman Cancer Institute, Salt Lake City, UT, USA; ${ }^{2}$ Department of Communication, University of Utah, Salt Lake City, UT, USA; ${ }^{3}$ National Cancer Institute, Bethesda, MD, USA; ${ }^{4}$ ICF, Rockville, MD, USA; ${ }^{5}$ National University of Singapore, Singapore, Singapore; ${ }^{6}$ Office of Public Health Genomics, Centers for Disease Control and Prevention, Atlanta, GA, USA. Correspondence: Kimberly A. Kaphingst (kim.kaphingst@hci.utah.edu)
} 


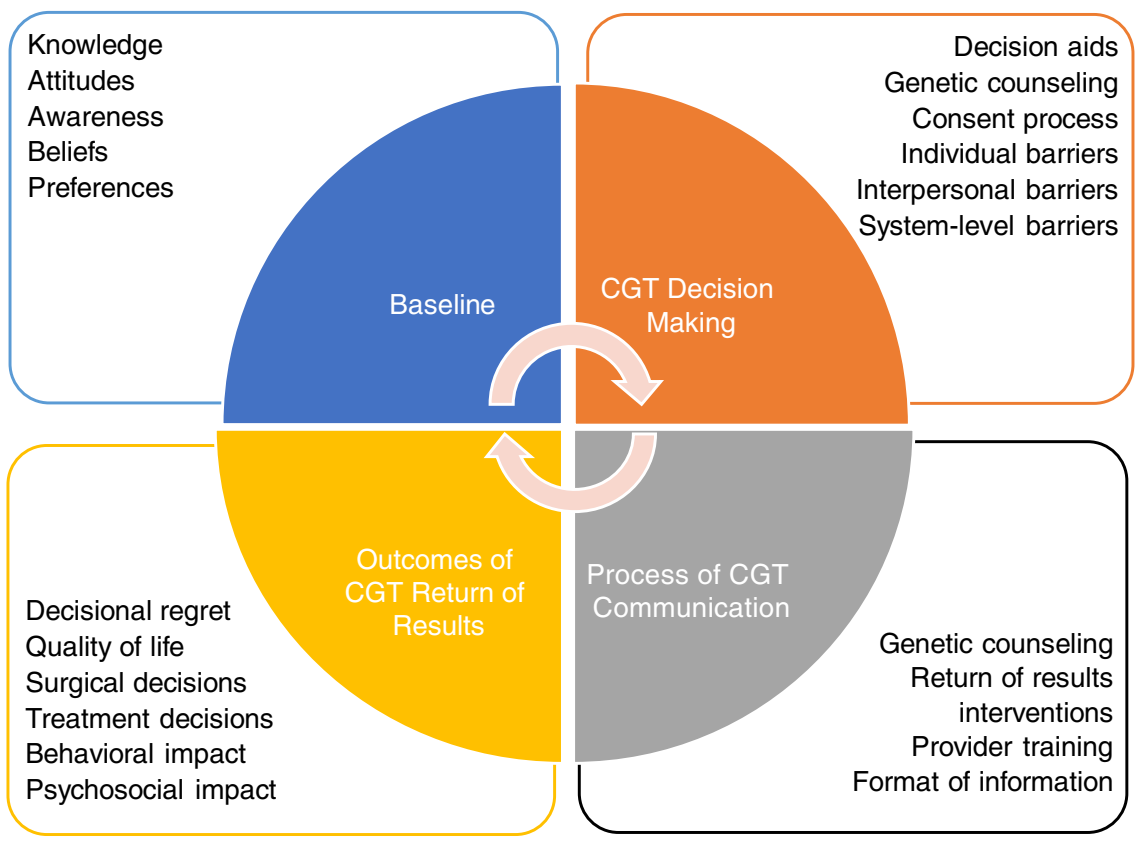

Fig. 1 Phases of cancer-related genetic and genomic testing (CGT) communication.

In our landscape analysis, we proposed a model delineating phases of CGT-related communication with patients, consumers, and the general public, which is based on models of patient-provider communication. ${ }^{14}$ As shown in Fig. 1, the phases of this continuum are (1) baseline knowledge and awareness of CGT, which forms the context for communication; (2) decision making (i.e., any communication about whether or not to have CGT); (3) process of communication about CGT (e.g., return of results); and (4) intermediate and distal outcomes of communication of CGT results (e.g., anxiety, health behavior change). ${ }^{13}$ As discussed above, prior reviews have mostly focused on the last phase. However, developing a complete understanding of communication outcomes requires a thorough analysis of all phases of the continuum.

The purpose of this review was to comprehensively examine published empirical research on communication about CGT between providers and patients or with consumers across the four phases of our continuum. Because our purpose was to describe the state of this research, we conducted a scoping review, a type of review that maps the relevant literature, ${ }^{15}$ rather than a systematic review focused on a more targeted question. We investigated the characteristics of studies examining the communication of CGT information, as well as the populations included in these studies, to identify both areas of strength and research gaps within the literature.

\section{Literature search}

We utilized Preferred Reporting Items for Systematic Reviews and Meta-Analysis (PRISMA) guidelines. ${ }^{16} \mathrm{We}$ conducted a comprehensive electronic literature search of articles published between January 2010 and January 2017 in the following databases: Medline, Embase, CINAHL, PsycINFO, Cochrane Library, and ERIC. This timeframe was selected both to allow a focus on recent research and to capture the period when next-generation sequencing technologies became available. We used controlled vocabulary (MeSH, EMTREE, and PsycInfo Subject Headings) where possible, combined with key words due to the lack of standard search terms in this literature. The broad categories for search terms included cancer, genetic/ genomic communication, provider/direct-to-consumer, and patient/public. The full Medline search strategy is presented in Supplemental Appendix A.

\section{Inclusion and exclusion criteria}

Detailed inclusion and exclusion criteria were developed through an iterative process of review and testing. Inclusion criteria were English language, included cancer-related genetic and/or genomic information, and relevant to at least one phase of the CGT communication. Articles were excluded if they focused only on providers or family communication, presented normative analyses, or did not present any empirical data. Articles were not restricted based on study design or outcome.

A total of 9243 unique papers were initially identified (Fig. 2). All titles and abstracts were reviewed by a trained coder, with $20 \%$ reviewed by a second coder. The level of agreement between coders for the decision to advance the paper to full-text review was adequate ${ }^{17}$ (Cohen's kappa $=$ 0.70 ; intercoder agreement $=92 \%$ ). For dual-coded articles, if at least one coder indicated "yes," the article progressed to the next stage of review. All full-text articles $(n=1297)$ were then evaluated by two independent coders; disagreements were 


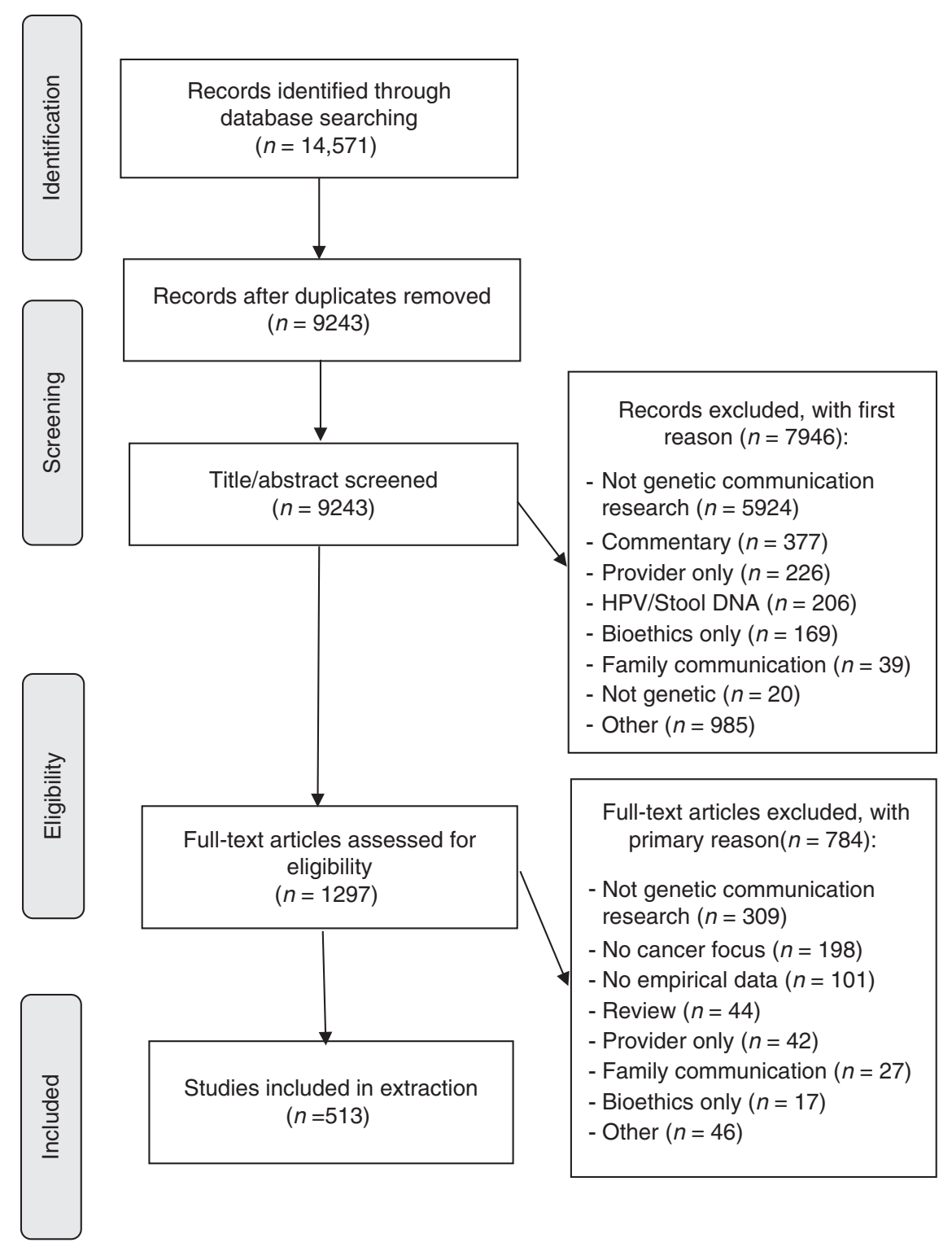

Fig. 2 Preferred Reporting Items for Systematic Reviews and Meta-Analysis (PRISMA) flow diagram. HPV human papilloma virus.

resolved by a third coder. There were 513 articles included in the final sample (Supplemental Table).

\section{Data extraction}

We developed a detailed data extraction protocol through several iterative rounds of review and testing (Supplemental Appendix B). The protocol included five major sections: (1) general study information, (2) quantitative outcomes and/or qualitative themes, (3) cancer and genetic area of focus, (4) participant characteristics, and (5) return of results processes. A single coder extracted information from each article, with a second coder extracting information for $10 \%$ of the articles. A third coder reconciled all discrepancies and determined a final code. Across 84 codes, only six had intercoder reliability that fell beneath predetermined thresholds (i.e., Cohen's kappa of
0.60 and $80 \%$ intercoder agreement). The consensus coder also reviewed the use of these six codes.

\section{RESULTS}

\section{Study characteristics}

Over half (57\%) of included studies were conducted in the United States (Table 1). A range of study designs was used; the majority of papers (54\%) utilized a quantitative, observational design (e.g., longitudinal, cross-sectional survey). The next most common study design was qualitative (e.g., interviews, focus groups; $23 \%$ ), whereas about $14 \%$ described randomized intervention trials or quasi-experiments (i.e., had a comparison group). The median sample size was 214 for quantitative, observational studies; 27 for qualitative studies; and 200 for intervention studies. 
Table 1 Characteristics of included studies $(n=513)$

\begin{tabular}{|c|c|c|}
\hline Characteristic & $n$ & $\%$ \\
\hline \multicolumn{3}{|l|}{ Countries $^{\mathrm{a}}$} \\
\hline United States & 292 & 57.0 \\
\hline The Netherlands & 51 & 10.0 \\
\hline United Kingdom & 31 & 6.0 \\
\hline Canada & 30 & 6.0 \\
\hline Other(s) & 121 & 23.3 \\
\hline \multicolumn{3}{|l|}{ Study designs } \\
\hline Quantitative, observational & 279 & 54.4 \\
\hline Qualitative & 120 & 23.4 \\
\hline Randomized intervention or quasi-experiment & 70 & 13.6 \\
\hline Mixed methods & 32 & 6.2 \\
\hline Case studies & 12 & 2.3 \\
\hline \multicolumn{3}{|c|}{ Cancer genetic or genomic (CGT) continuum phase ${ }^{a}$} \\
\hline Baseline knowledge and awareness & 160 & 31.1 \\
\hline Decision making about uptake of CGT & 112 & 21.8 \\
\hline Process of CGT communication & 106 & 20.7 \\
\hline Outcomes of CGT results disclosure & 243 & 47.3 \\
\hline Cancer only condition studied & 421 & 82.1 \\
\hline \multicolumn{3}{|l|}{ Cancer type $\mathrm{a}^{\mathrm{a}}$} \\
\hline Breast and/or ovarian cancer & 276 & 53.8 \\
\hline Cancer-general & 73 & 14.2 \\
\hline Multiple cancers or cancer syndromes & 55 & 10.7 \\
\hline Colorectal cancer & 51 & 10.0 \\
\hline \multicolumn{3}{|l|}{ Type of CGT information ${ }^{a}$} \\
\hline$B R C A 1 / B R C A 2$ & 261 & 50.9 \\
\hline Lynch syndrome/HNPCC & 40 & 7.8 \\
\hline Exome or genome sequencing & 30 & 5.8 \\
\hline SNP/polygenic risk score & 19 & 3.7 \\
\hline Gene panel/multigene panel & 9 & 1.7 \\
\hline p53/Li-Fraumeni & 7 & 1.4 \\
\hline p16 & 7 & 1.4 \\
\hline Pharmacogenetic/pharmacogenomic & 3 & 0.6 \\
\hline Hypothetical information used & 115 & 22.4 \\
\hline Direct-to-consumer information used & 34 & 6.6 \\
\hline
\end{tabular}

\section{CGT continuum phases}

With regard to phases of the CGT continuum, which were not mutually exclusive, the most common focus was on outcomes of CGT results disclosure (47\%; e.g., ref. ${ }^{18}$ ). About $31 \%$ of studies focused on examining baseline CGT knowledge and awareness (e.g., ref. ${ }^{19}$ ). Approximately equal proportions of studies focused on decision making about CGT uptake $(22 \%$; e.g., ref. ${ }^{20}$ ) and the process of CGT communication (e.g., return of results; $21 \%$; see ref. ${ }^{21}$ ). Illustrative examples of studies in each CGT continuum phase, organized by type of study design, are provided in Supplemental Box 1.

\section{Outcomes assessed}

Figure 3 shows study quantitative outcomes/qualitative themes by CGT continuum phase, organized by study design.
Psychosocial outcomes/themes (e.g., anxiety, distress) were most common. In randomized trials and quasi-experiments (Fig. 3a) and quantitative, observational studies (Fig. 3b), decision making outcomes/themes were more commonly examined than communication outcomes/themes. Behavioral outcomes/themes (e.g., health behaviors, behavioral intentions) were the least common category for all study designs except quantitative observational designs, for which patientreported outcomes/themes were less common (Fig. 3b).

\section{Cancer and CGT focus}

The vast majority of included papers focused on genetic and genomic information related to cancer only (82\%; Table 1). About one-quarter (22\%) analyzed responses to hypothetical CGT information. Of the studies presenting hypothetical information, the majority (53\%) examined baseline CGT knowledge and awareness. The most common types of cancer studied were breast and/or ovarian (54\%), followed by colorectal cancer (10\%). Consistent with this, the most common type of genetic information discussed was BRCA1/ BRCA2 genes (51\%), which affect risk of breast and ovarian cancer. The proportion of papers in each phase discussing BRCA $1 / 2$ testing was $39 \%$ for baseline knowledge and awareness of CGT, $51 \%$ for decision making about uptake of CGT, 53\% for process of communication about CGT, and $60 \%$ for outcomes of communication of CGT results. Only $8 \%$ of papers focused on Lynch syndrome, also known as hereditary nonpolyposis colorectal cancer (HNPCC). Few papers examined information generated through direct-toconsumer genetic testing (7\%).

\section{Next-generation technologies}

Relatively few papers focused on genomic information generated by exome or genome sequencing $(5 \%)$ or multigene panels (2\%). Of these papers, $17 \%$ related to baseline knowledge and awareness of these technologies, $33 \%$ to decision making about uptake of sequencing or multigene panels, and $27 \%$ to the process and $23 \%$ to the outcomes of communication of test results. Similar to the overall sample, the most common type of outcome in these papers was psychosocial (74\% of papers), followed by decision making (49\%). Few papers assessed communication (18\%) or behavioral $(10 \%)$ outcomes.

\section{Characteristics of study participants}

The most commonly reported characteristics of study participants were sex (reported in $93 \%$ of studies) and age (reported in $88 \%$; Table 2). In almost half of studies (45\%), all participants were women. In contrast, only $1 \%$ of studies had all male participants. Few studies focused on younger adults; $7 \%$ of studies included participants with a mean age of 35 years or younger. In addition, few studies included pediatric populations; only $5 \%$ of studies included minors under 18 years of age. Only $7 \%$ of studies recruited participants based on their parental status. Few studies (3\%) reported whether or not participants were from rural/frontier areas. Reporting of 
a

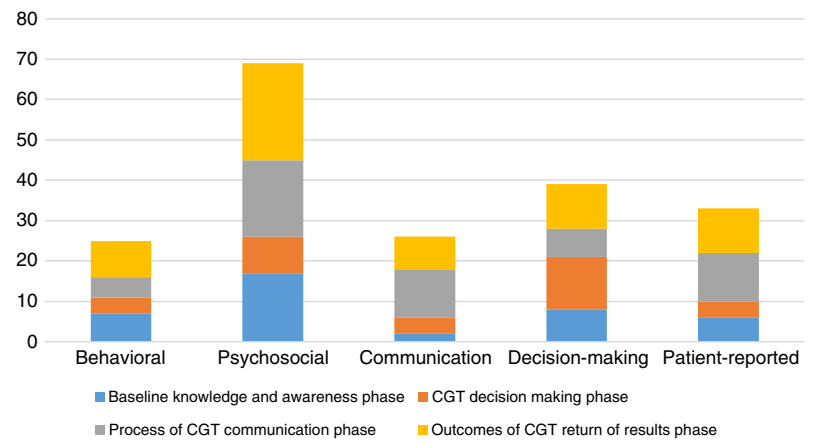

b

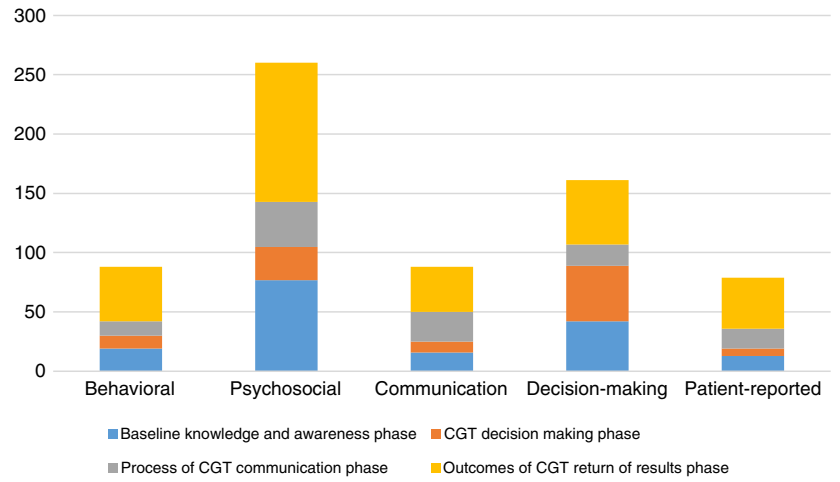

C 140

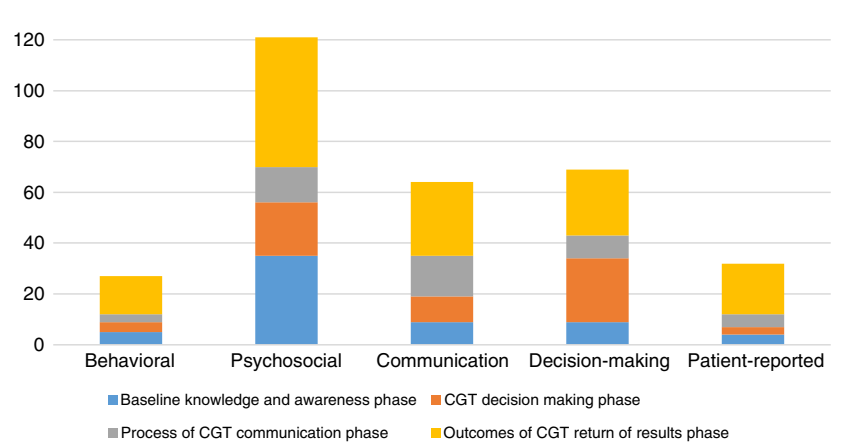

d

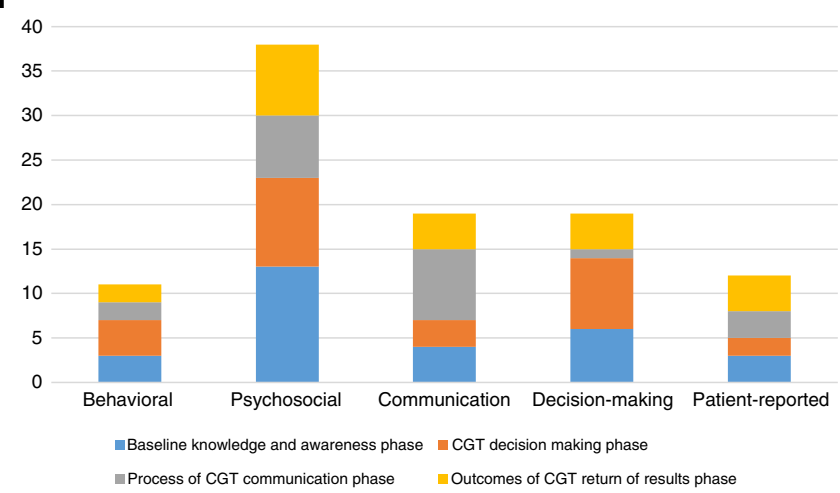

Fig. 3 Outcome and theme categories by study design and phase of cancer-related genetic and genomic testing (CGT) continuum. (a) Outcome categories for randomized trials and quasi-experiments $(n=70)$. (b) Outcome categories for quantitative observational studies $(n=279)$. (c) Theme categories for qualitative studies $(n=120)$. (d) Outcome and theme categories for mixed methods studies $(n=32)$. Outcome and theme categories not mutually exclusive; $y$-axes represent the number of studies for each type of study design.

socioeconomic status of participants was variable. Although $70 \%$ of studies reported on participants' educational attainment, only $28 \%$ reported on income. Among the US studies that reported educational data, only $2 \%$ met our a priori threshold of having a sample with $70 \%$ of participants with a high school education or less.

The race and/or ethnicity of study participants was reported in about half of the studies (54\%); most US studies (86\%) reported these data. Among studies that reported race/ ethnicity, we examined what proportion had $70 \%$ or more of participants from a specific racial or ethnic group. In total, $63 \%$ of studies met this threshold for White/Caucasian, $5 \%$ for Black/African American, $4 \%$ for Hispanic/Latino, $1 \%$ for Asian/Pacific Islander, and $0.4 \%$ for Native American/Alaska Native. We also found that $17 \%$ of included studies had no White/Caucasian participants, $52 \%$ had no Black/African American participants, $65 \%$ had no Hispanic/Latino participants, 72\% had no Asian/Pacific Islander participants, and $87 \%$ had no Native American/Alaska Native participants.

\section{DISCUSSION}

This scoping review examined the characteristics of more than 500 recently published papers focused on the communication of CGT information. Although a range of study designs have been employed in this area of research, the majority of papers utilized an observational quantitative design. This finding suggests a need for intervention trials to examine the effects of different communication formats, channels, and strategies in presenting genetic and genomic information to the public, informing shared decision making about testing, and enabling accessible and patient-centered approaches to returning genetic test results to patients and their families. With respect to the CGT continuum phases, the review also showed that there has been more attention to the outcomes of CGT results disclosure than to decision making regarding testing or the process of results disclosure. At present, studies that examine the outcomes of results disclosure often do not examine the communication process (e.g., what, how, when, and by whom results are delivered) in detail. The field needs a more fine-grained understanding of the effects of the communication processes and contexts on the intermediate and distal outcomes of CGT results disclosure.

Our findings also show a continuing emphasis on psychosocial outcomes in this literature. This finding is consistent with older reviews showing a focus on anxiety and distress outcomes following return of genetic test results. ${ }^{8-10}$ Although emotional and cognitive psychosocial outcomes are certainly important, the literature needs to expand. Models of patient-provider communication $^{14}$ and health behavior 
Table 2 Characteristics of participants in included studies $(n=513)$

Characteristic n $\quad \%$

\begin{tabular}{|c|c|c|}
\hline \multicolumn{3}{|l|}{ Age $(n=478)^{a}$} \\
\hline Mean age 35 years or younger & 34 & $7.1^{\mathrm{b}}$ \\
\hline Minors less than 18 included & 24 & $5.0^{b}$ \\
\hline Parents $(n=513)$ & 35 & 6.8 \\
\hline \multicolumn{3}{|l|}{$\operatorname{Sex}(n=479)^{a}$} \\
\hline $100 \%$ women & 227 & $47.3^{c}$ \\
\hline $100 \%$ men & 7 & $1.4^{c}$ \\
\hline$\geq 25 \%$ men & 179 & $37.3^{c}$ \\
\hline \multicolumn{3}{|l|}{ Race/ethnicity $(n=279)^{a}$} \\
\hline$\geq 70 \%$ White & 177 & $63.4^{d}$ \\
\hline $0 \%$ White & 47 & $16.8^{d}$ \\
\hline$\geq 70 \%$ Black/African American & 13 & $4.6^{\mathrm{d}}$ \\
\hline 0\% Black/African American & 144 & $51.6^{d}$ \\
\hline$\geq 70 \%$ Hispanic/Latino & 10 & $3.6^{d}$ \\
\hline 0\% Hispanic/Latino & 181 & $64.9^{d}$ \\
\hline$\geq 70 \%$ Asian/Pacific Islander & 3 & $1.1^{d}$ \\
\hline 0\% Asian/Pacific Islander & 202 & $72.4^{d}$ \\
\hline$\geq 70 \%$ Native American/Alaska Native & 1 & $0.4^{d}$ \\
\hline $0 \%$ Native American/Alaska Native & 244 & $87.4^{d}$ \\
\hline \multicolumn{3}{|l|}{ Ashkenazi Jewish $(n=49)$} \\
\hline 100\% Ashkenazi Jewish & 10 & $20.4^{e}$ \\
\hline Income reported & 145 & 28.3 \\
\hline Education reported & 360 & 70.2 \\
\hline Education reported for US studies & 208 & \\
\hline$\geq 70 \%$ had high school education or less & 4 & $1.9^{f}$ \\
\hline$\geq 70 \%$ had some college or greater & 140 & $67.3^{f}$ \\
\hline Rurality reported & 14 & 2.7 \\
\hline \multicolumn{3}{|c|}{$\begin{array}{l}\text { aNot mutually exclusive. } \\
\text { bPercentage of } 478 \text { studies reporting age data. } \\
\text { cPercentage of } 479 \text { studies reporting sex of participants. } \\
\text { dPercentage of } 279 \text { studies reporting race/ethnicity of participants. } \\
\text { ePercentage of } 49 \text { studies reporting whether or not participants were Ashkenaz } \\
\text { Jewish. } \\
\text { Percentage of US-based studies. }\end{array}$} \\
\hline
\end{tabular}

change suggest that behavioral, decision making, clinical communication, patient-reported, and psychosocial outcomes are all critical to investigate to improve patient health outcomes. Additional research is needed to understand under what circumstances, in which contexts, and in what populations, disclosure of genetic and genomic information affects health behavior change. A greater focus on outcomes such as health behaviors and medical decisions, in concert with more in-depth examination of the processes of return of results communication, can shed further light on the contexts in which CGT information may affect behavior change.

The review also showed a strong focus on breast and ovarian cancer and $B R C A 1 / 2$ in this literature. In contrast, relatively few papers focused on Lynch syndrome, despite the longstanding availability of this testing, strength of evidence for the clinical importance of testing for genes associated with Lynch syndrome, and the growth of universal screening programs for patients diagnosed with colorectal and endometrial cancer. ${ }^{22-24}$ This is a clear gap for which additional research is needed. ${ }^{25}$ In addition, despite the rapidly growing importance of next-generation technologies such as multigene panel tests and genome sequencing, ${ }^{3,26-29}$ relatively few studies to date have focused on communication of results generated by these technologies. Although tests for hereditary breast and ovarian cancer and Lynch syndrome have been available longer and currently have the strongest evidence for return of results in clinical settings, ${ }^{30}$ it is important for researchers investigating CGT communication processes to conduct studies as soon as possible to inform how to effectively return results from next-generation technologies that are being introduced into clinics so that the results of communication research are clinically relevant in the era of precision medicine. Particularly given the greater complexity, larger amount of information provided, and greater possibility of uncertainty and ambiguity inherent in such results, ${ }^{26,27,29,31,32}$ future research is needed that investigates the process and outcomes of communication of these results and, when examining outcomes, moves beyond psychosocial outcomes to examine how such information may affect behavior and how patients communicate about the results with others. Furthermore, although our prior landscape analysis identified a substantial number of prior reviews investigating direct-to-consumer genetic testing, ${ }^{13}$ relatively few empirical studies included in this scoping review did so. Studies investigating this increasingly important phenomenon are needed to understand CGT communication with patients and the public that occurs without a provider as a learned intermediary. ${ }^{33}$

Finally, the findings from this scoping review highlight the strong need to diversify the population subgroups included in studies of CGT communication. Reporting of demographic factors should be improved to better characterize study samples. However, the participant data that have been reported showed important gaps in inclusion. Age and sex were most commonly reported, and these data showed the need for increasing research with pediatric and young adult populations and with men. Prior reviews have documented persistent barriers to access and use of genetic and genomic information by minority racial and ethnic groups. ${ }^{34}$ Only about half of the included studies reported on the race/ ethnicity of participants, making it more difficult to examine racial and ethnic disparities. The data reported revealed a clear lack of inclusion of participants from racial and ethnic groups other than Caucasians. Aspects of socioeconomic status such as income were rarely reported, making it difficult to examine disparities. Reported educational data showed that very few studies focused on samples with lower levels of education. Although the limited inclusion of individuals from minority racial and ethnic groups has been noted previously, our scoping review also highlights lack of inclusion for those with limited educational attainment and for pediatric and young adult populations. The findings also identify lack of inclusion of participants from minority racial and ethnic groups as a consistent and pervasive problem throughout the 
literature on CGT communication. As the cost of genomic technologies decreases and these technologies become more accessible, guidance is needed regarding effective and tailored communication approaches across population subgroups. ${ }^{34,35}$ The issue of inclusion in CGT communication research is particularly pressing given the goals of precision medicine initiatives such as the All of Us Research Program to reach diverse populations.

The results of this review should be considered in light of its limitations. The lack of standard MeSH headings and key words in this literature made it difficult to locate relevant studies. Establishing consistent key terms for CGT communication processes (e.g., genetic communication, cancer) would facilitate future reviews. Because of our interest in describing the characteristics of the peer-reviewed literature, unpublished studies were not included. Studies from different countries described race/ethnicity and socioeconomic status differently; we focused on describing results from the United States that were more likely to report such characteristics. Because this is a scoping review, we were not able to synthesize the findings to generate overall effect sizes or summarize the effect of a specific communication process, as is done with a systematic review or meta-analysis. Our aims were to present a map of the literature on CGT communication and identify important research gaps; future reviews can home in on a subset of studies to investigate a particular research question in detail.

In summary, this scoping review highlights areas of strength in recent literature on communication of CGT information. In particular, much is known regarding communication about the genes $B R C A 1 / 2$, and psychosocial outcomes of testing have been a strong area of emphasis. Research gaps were also identified, including the need for additional studies on communication processes, expanding the types of outcomes studied, increased research related to Lynch syndrome and next-generation technologies, and inclusion of diverse samples with complete descriptions of participant characteristics. Addressing these research gaps can help improve the health of cancer patients and those at risk of cancer, and reduce disparities in access to and use of CGT across population subgroups.

\section{SUPPLEMENTARY INFORMATION}

The online version of this article (https://doi.org/10.1038/s41436018-0402-0) contains supplementary material, which is available to authorized users.

\section{ACKNOWLEDGEMENTS}

Financial support was provided by the National Cancer Institute's Behavioral Research Program through HHSN261201700078P. We also acknowledge the direct financial support for the research reported in this publication provided by the Huntsman Cancer Foundation. We would like to acknowledge assistance from the Clinical and Translational Science Award (CTSA) Systematic Review Core at the University of Utah. We also thank Courtney Tern and Angela Falisi for their work as coders, and Bradford
Hesse and Charlisse Caga-Anan for their invaluable contributions to the study design. The content is solely the responsibility of the authors and does not necessarily represent the official views of the National Institutes of Health $(\mathrm{NIH})$.

\section{DISCLOSURE}

The authors declare no conflicts of interest.

\section{REFERENCES}

1. Kensler TW, Spira A, Garber JE, Szabo E, Lee JJ, Dong Z. Transforming cancer prevention through precision medicine and immune-oncology. Cancer Prev Res. 2016;9:2-10.

2. Judkins T, Leclair B, Bowles $K$, et al. Development and analytical validation of a 25-gene next generation sequencing panel that includes the BRCA1 and BRCA2 genes to assess hereditary cancer risk. BMC Cancer. 2015;15:215.

3. Hall MJ, Forman AD, Pilarski R, Wiesner G, Giri VN. Gene panel testing for inherited cancer risk. J Natl Compr Canc Netw. 2014;12:1339-1346.

4. Hiraki S, Rinella ES, Schnabel F, Oratz R, Ostrer H. Cancer risk assessment using genetic panel testing: considerations for clinical application. J Genet Couns. 2014;23:604-617.

5. Beery TA, Williams JK. Risk reduction and health promotion behaviors following genetic testing for adult-onset disorders. Genet Test. 2007;11:111-123.

6. Burke W, Psaty BM. Personalized medicine in the era of genomics. JAMA. 2007;298:1682-1684.

7. Garraway LA, Verweij J, Ballman KV. Precision oncology: an overview. J Clin Oncol. 2013;31:1803-1805.

8. Heshka JT, Palleschi C, Howley H, Wilson B, Wells PS. A systematic review of perceived risks, psychological and behavioral impacts of genetic testing. Genet Med. 2008;10:19-32.

9. Butow PN, Lobb EA, Meiser B, Barratt A, Tucker KM. Psychological outcomes and risk perception after genetic testing and counselling in breast cancer: a systematic review. Med J Aust. 2003;178:77-81.

10. Kaphingst KA, McBride CM. Patient responses to genetic information: studies of patients with hereditary cancer syndromes identify issues for use of genetic testing in nephrology practice. Semin Nephrol. 2010;30:203-214.

11. Hollands GJ, French DP, Griffin SJ. The impact of communicating genetic risks of disease on risk-reducing health behaviour. BMJ. 2016;352:1/2p.

12. McBride CM, Wade CH, Kaphingst KA. Consumers' views of direct-toconsumer genetic information. Ann Rev Genomics Hum Genet. 2010;11:427-446.

13. Peterson E, Chou W-YS, Gaysynsky A, et al. Communication of cancerrelated genetic and genomic information: a landscape analysis of reviews. Transl Behav Med. 2018:8:59-70.

14. Epstein RM, Street RL. Patient-centered communication in cancer care: promoting healing and reducing suffering. Bethesda, MD: National Cancer Institute; 2007. P.

15. Arksey H, O'Malley L. Scoping studies: towards a methodological framework. Int J Soc Res Methodol. 2005;8:19-32.

16. Moher D, Liberati A, Tetzlaff J, Altman DG, The PRISMA Group. Preferred reporting items for systematic reviews and meta-analyses: the PRISMA statement. PLoS Med. 2009;6:e1000097.

17. Fleiss J, Levin B, Paik M. Statistical methods for rates and proportions. 3rd ed. New York: John Wiley \& Sons; 2003.

18. Aktan-Collan $\mathrm{K}$, Kaariainen $\mathrm{H}$, Jarvinen $\mathrm{H}$, et al. Psychosocial consequences of predictive genetic testing for Lynch syndrome and associations to surveillance behaviour in a 7-year follow-up study. Fam Cancer. 2013;12:639-646.

19. Agurs-Collins T, Ferrer R, Ottenbacher A, Waters EA, O'Connell ME, Hamilton JG. Public awareness of direct-to-consumer genetic tests: findings from the 2013 U.S. Health Information National Trends Survey. J Cancer Educ. 2015;30:799-807.

20. Alderfer MA, Zelley K, Lindell RB, et al. Parent decision-making around the genetic testing of children for germline TP53 mutations. Cancer. 2015;121:286-293.

21. Thomassen G, Sarangi S. Evidence-based familial risk explanations in cancer genetic counselling. Health Risk Soc. 2012;14:607-626. 
22. Vindigni S, Kaz A. Universal screening of colorectal cancers for Lynch syndrome: challenges and opportunities. Dig Dis Sci. 2016;61:969-976.

23. Hampel $\mathrm{H}$. Point: justification for Lynch syndrome screening among all patients with newly diagnosed colorectal cancer. J Natl Compr Canc Netw. 2010;8:597-601.

24. Provenzale D, Gupta S, Ahnen DJ, et al. Genetic/familial high-risk assessment: colorectal version 1.2016, NCCN clinical practice guidelines in oncology. J Natl Compr Canc Netw. 2016;14:1010-1030.

25. Slater MD, Long M, Bettinghaus EP, Reineke JB. News coverage of cancer in the United States: a national sample of newspapers, television, and magazines. J Health Commun. 2008;13:523-527.

26. Domchek SM, Bradbury A, Garber JE, Offit K, Robson ME. Multiplex genetic testing for cancer susceptibility: out on the high wire without a net? J Clin Oncol. 2013;31:1267-1270.

27. Society of Gynecologic Oncology. SGO clinical practice statement: next generation cancer gene panels versus gene by gene testing. 2014 https://www.sgo.org/clinical-practice/guidelines/next-generation-cancergene-panels-versus-gene-by-gene-testing/. Accessed 3 May 2018.

28. Easton DF, Pharoah PDP, Antoniou AC, et al. Gene-panel sequencing and the prediction of breast-cancer risk. N Engl J Med. 2015; $372: 2243-2257$
29. Wolfe Schneider K, Anguiano A, Axell L, et al. Collaboration of Colorado cancer genetic counselors to integrate next generation sequencing panels into clinical practice. J Genet Couns. 2014;23:640-646.

30. Khoury MJ, Bowen MS, Clyne M, et al. From public health genomics to precision public health: a 20-year journey. Genet Med. 2018;20:574-582.

31. Slavin TP, Niell-Swiller M, Solomon I, et al. Clinical application of multigene panels: challenges of next-generation counseling and cancer risk management. Front Oncol. 2015;5:208.

32. Mills R, Haga SB. Genomic counseling: next generation counseling. Genet Couns. 2014;23:689-692.

33. US Food and Drug Administration. FDA authorizes, with special controls, direct-to-consumer test that reports three mutations in the BRCA breast cancer genes. 2018. https://www.fda.gov/NewsEvents/Newsroom/ PressAnnouncements/ucm599560.htm. Accessed 7 May 2018.

34. Kaphingst KA, Goodman MS. Importance of race and ethnicity in individuals' use of and responses to genomic information. Pers Med. 2016;13:1-4.

35. McBride CM, Bowen D, Brody LC, et al. Future health applications of genomics: priorities for communication, behavioral, and social sciences research. Am J Prev Med. 2010;38:561-566. 